\title{
On the Context Dependence of Many
}

\author{
Matthias F.J. Hofer* \\ Vienna University of Technology
}

\begin{abstract}
We augment the applicability of Lappin's intensional parametrization of the determiners many and few by combinatorial means, and show how to arrive at graded interpretations of corresponding natural language statements.
\end{abstract}

Keywords natural language semantics; vagueness; quantifiers; fuzzy logic

\section{Introduction}

Our aim in this paper is incorporate combinatorial techniques into natural language semantics, and, particularly, to develop a concept of natural language quantification. During the last decades, there have been several approaches to context dependent interpretation regarding determiners like many, and we find that they can be divided into two main streams. One is carried out by linguists, like Fernando and Kamp [6], Keenan and Stavi [9], Barwise and Cooper [1], or Westerståhl [13,14], and Lappin [10,11,12], and they all share the feature of twovalued interpretation, that is, the expressions, involving many, are either true or false. Then, there is the other community, namely the fuzzy logicians, like Zadeh [15], Glöckner [8], or Hájek [2], and Fermüller [3,4,5]. They, accept the need of graded interpretations, while the use of contexts, that is in particular also the acceptance of intensional aspects of the matter, is still best observed in [12], where extensionality and intensionality get fit into one unifying frame. In both, the extensional and the intensional setting, one can make use of comparison classes. Lappin calls them comparison sets in the extensional case and normative situations for the intensional case. Even though we follow his terminology, we intend to show, that the underlying combinatorial pattern is the same in both cases. In so doing, we will give four additional extensional readings for many in Section 2, and eight new intensional readings in Section 3, and each time emphasize the combinatorial underpinnings. Also, in Section 2, we characterize what can be seen as a canonical structure for contexts, which we call a distinction tree. The ideas get illustrated through an easy context dependent statement, involving many. Eventually, in Section 4, we show how one can go down the road to graded interpretations of many, and how many and few are functionally related. Thus, this paper aims at bringing the two streams closer together.

\footnotetext{
* Supported by Austrian Science Fund (FWF) I1897-N25 (MoVaQ)
} 


\section{Lappin's approach and beyond}

Our main reference throughout this paper will be Shalom Lappin's article [12], and particularly his two-valued intensional parametric interpretation of $\|$ many $\|$, namely this one:

$$
\begin{aligned}
& \|B\|^{\text {sa }} \in \| \text { many } \|\left(\|A\|^{\text {sa }}\right) \text { iff } \\
& \qquad S \neq \emptyset, \text { and for all sn } \in S, \quad\|\| A\left\|^{\text {sa }} \cap\right\| B\left\|^{\text {sa }}\right\| \geqslant\|\| A\left\|^{\text {sn }} \cap\right\| B\left\|^{\text {sn }}\right\|
\end{aligned}
$$

The statement is formulated in the following notation (cf. [12], p. 601):

- sa is an actual situation (a situation that supports only states of affairs that we identify as actual).

- $S$ is a set of normative situations sn.

- a situation is, effectively, a non-maximal possible world. ${ }^{1}$

- for a predicate $P$, we have $\|P\|^{s}=\{a: s \vDash P(a)\}$, thus the set of $a$ such that $P(a)$ holds in $s .^{2}$

Thus, assuming we have a domain of objects, that share two crisp $^{3}$ properties $A$ and $B$, and distinguished other domains where all objects have the same two properties, Lappin's semantics of many evaluates to true iff the original domain is at least as big, in terms of cardinality, as the distinguished other domains. Hence, it is necessary to point out that not the range of the determiner is vague, but what is meant by vague, is that there are different possibilities for interpreting it. Contrasting it with $\|$ every $\|$, Lappin states this:

"By contrast || many || and || few || allow a large number of distinct interpretations whose specification involves essential reference to contextual parameters. In fact, these quantifiers seem to be vague in a way that GQ's like \| every are not." ( [12], p. 600)

In this section, we will follow that strategy, and come back to the possibility of augmenting it in Section 4. The following statement of Lappin's can be seen as a motivation for his approach:

"The determiners many and few are problematic for generalized quantifier theory because, as has frequently been noted, their interpretations are radically context-dependent and under-determined." ( [12], p. 599)

\footnotetext{
${ }^{1}$ Cf. p. 601 in [12], footnote 6

${ }^{2}$ Cf. p. 602 in [12], footnote 8

${ }^{3}$ Here, crisp means two-valued
} 
He singles out the two options of extensional readings and intensional readings, but in each case (1) is meant to give an adequate semantics. Referring to the work of Keenan and Stavi (1986, [9]) and Fernando and Kamp (1996, [6]) in this area, he gives the following assessment:

"Both types of analysis are problematic in that either they do not allow for certain readings of many (few), or they generate multiple ambiguity with no apparent upper bound on the set of possible interpretations for these determiners. In Section 2 I present a different sort of intensional account of vague quantifiers which avoids these difficulties." ( [12], p. 601)

Also, he explains how extensionality and intensionality are related:

"In addition to intensional readings like those indicated for (7) and (9), it is possible to derive from $(5)^{4}$ the full range of extensional readings which have been proposed on alternative accounts of many by extensionalising $S$ through the requirement that the elements of $S=$ sa."( [12], p. 603)

For illustration, Lappin gives nine different extensional readings of many, referred to as (10), (11a-d), and (12a-d) (in [12]), and embeds them into his setting. This appears to be a neat formal frame for figuring things. Along with it, we present four additional interpretations that fit into this frame, to extend his list of nine into a list of 13 . We want to employ several "comparison sets" to augment applicability. Thus, for $i \in\{1, \ldots, k\}$ let $C_{i}$ be the $i$-th comparison set. Also, we define:

$p_{B}^{\mathrm{sa}}:=\left|\|A\|^{\mathrm{sa}} \cap\|B\|^{\mathrm{sa}}\right|$, and $p_{i}^{\mathrm{sa}}:=\left|\|A\|^{\mathrm{sa}} \cap\left\|C_{i}\right\|^{\mathrm{sa}}\right|$.

Be $I_{a}:=\left\{i: i \in\{1, \ldots, k\}, p_{i}^{\mathrm{sa}}>p_{B}^{\mathrm{sa}}\right\} \quad$, and $a:=\left|I_{a}\right|$.

Be $I_{b}:=\left\{i: i \in\{1, \ldots, k\}, p_{i}^{\mathrm{sa}}=p_{B}^{\mathrm{sa}}\right\} \quad$, and $b:=\left|I_{b}\right|$.

Be $I_{c}:=\left\{i: i \in\{1, \ldots, k\}, p_{i}^{\mathrm{sa}}<p_{B}^{\mathrm{sa}}\right\} \quad$, and $c:=\left|I_{c}\right|$.

$\left|C^{+}\right|:=\max \left\{\left|\left\|C_{i}\right\|^{\mathrm{sa}} \cap\|A\|^{\mathrm{sa}}\right|: i \in\{1, \ldots, k\}\right\}$

Now we can state our four additional interpretations for many:

(A1) $S=\left\{\mathrm{sn}: \mathrm{sn}=\mathrm{sa} \& p_{B}^{\mathrm{sa}} \geqslant p_{i}^{\mathrm{sa}}\right.$, for all $\left.i \in\{1, \ldots, k\}\right\}$

(A2) $S=\left\{\mathrm{sn}: \mathrm{sn}=\mathrm{sa} \& p_{B}^{\mathrm{sa}} \geqslant p_{i}^{\mathrm{sa}}\right.$, for most $\left.i \in\{1, \ldots, k\}\right\}$

(A3) $S=\left\{\mathrm{sn}: \mathrm{sn}=\mathrm{sa} \& \sum_{i \in I_{a}} p_{i}^{\mathrm{sa}}<\sum_{i \in I_{c}} p_{i}^{\mathrm{sa}}\right\}$

(A4) $S=\left\{\mathrm{sn}: \mathrm{sn}=\mathrm{sa} \&\left|C^{+}\right|<p_{B}^{\text {sa }}\right\}$

4 The (1) from the present paper is referred to as (5) in [12] 
The second one is clearly just a weakening of the first. Employing a '>'-sign, instead of the ' $\geqslant$ '-sign (in (A1) or (A2)), would be a strengthening. We will come back to this in a bit. The third one is apparently somewhat new, even though the meaning should be clear from a philosophical viewpoint. Having several comparison sets, it says the following: If the sum of the masses of the comparison sets smaller than the one under investigation, is bigger than the corresponding sum of the masses of the comparison sets bigger than the one under investigation, then $\|B\|^{\text {sa }} \in \|$ many $\|\left(\|A\|^{\text {sa }}\right)$ can be regarded adequate. The last one can be seen as a strengthening with respect to Lappin's 12a, using several, instead of one, comparison set. One can also think of very different readings of many. For example, there may be psychological reasons to consider something as many. In cases in which there are no comparison classes, or at least, they are not obvious, we can think of an information overflow interpretation. For example, if we look at a table, that hosts exactly five coins, most of us will be able to recognize the number of coins at once, without counting them. If we put 17 coins instead, most human brains will consider that as to many to handle at once, and will need to count them. It seems interesting to encounter such readings as well, but for the sake of brevity we will not so do here. One example we consider here informally, builds upon the idea of small children playing LEGO. What could bring a child to consider the number of red LEGO-bricks as many, is that there are more of the red sort than of all other colors. That would be Lappin's interpretation, if he had introduced more than one allowed comparison set, since we are in the extensional case. Still, it makes sense to talk about 'many red LEGO-bricks', even if there is a color of which there are more LEGO-bricks. That is what we formalize here. The intensional case would be the one of one child playing LEGO, but, e.g., at different days, and in different rooms, and using these different information clusters as comparison classes. This shall, on empirical grounds, motivate the need to extend Lappin's approach. The next example will be our running example for the remaining part of the paper. It is a context dependent statement that will be revisited in Section 3:

\section{"Many students are German"}

For a first assessment of this, we intend to use a situation sa to which we informally refer to as Vienna. Formally, we will define sa as follows:

Be $E$ the set of all people registered in Vienna. Thus, $E=\left\{p_{1}, \ldots, p_{m}\right\}$ for some $m \in \mathbb{N}$. We assume that 'being a student' is a crisp predicate, and is decidable, for all people living in Vienna. Hence, we separate the elements of $E$ in the following way:

$$
J_{\mathrm{st}}:=\left\{j: j \in\{1, \ldots, m\} \text { and } \operatorname{student}\left(p_{j}\right) \text { is true }\right\}
$$

Again, we assume that, for all such students, it is decidable whether one is German or not, thus we separate $I_{\mathrm{st}}$ further:

$$
J_{\mathrm{st}, G}:=\left\{j: j \in J_{\mathrm{st}} \text { and } \operatorname{German}\left(p_{j}\right) \text { is true }\right\}
$$

Similarly, assuming there are $k$ other nationalities $C_{1}, \ldots, C_{k}$, apart from the Austrians themselves, and that each person only possesses one citizenship, we have: 


$$
\begin{aligned}
& J_{\mathrm{st}, \mathrm{At}}:=\left\{j: j \in J_{\mathrm{st}} \text { and } \operatorname{Austrian}\left(p_{j}\right) \text { is true }\right\} \\
& J_{\mathrm{st}, C_{i}}:=\left\{j: j \in J_{\mathrm{st}} \text { and } C_{i}\left(p_{j}\right) \text { is true }\right\} \text { for all } C_{i} \text { with } i \in\{1, \ldots, k\} .
\end{aligned}
$$

Thus: $J_{\mathrm{st}}=J_{\mathrm{st}, G} \dot{\cup} J_{\mathrm{st}, \mathrm{At}} \dot{\cup} J_{\mathrm{st}, C_{1}} \dot{\cup} \ldots \dot{\cup} J_{\mathrm{st}, C_{k}}$

Before we continue presenting the example for the extensional assessment of (2), using only one situation, namely sa (= Vienna), we capture that context structure. Here, it is crucial, that the used predicates are crisp, and fulfill the law of excluded middle with respect to the root-set $E$ :

This intuitive picture captures basically the idea of a canonical structure for figuring orderings of objects in a predicative way: At each node, we add a new predicate, that all the node following objects have to fulfill forever. Thus, the leaves carry objects, that fulfill all the predicates of the preceding nodes. This tangible way of writing things down, can eventually lead to an increase of processability by machines. Lets give a definition:

Definition 1 (Distinction Tree). Be L a finite list of objects, and $T$ a finitely branching (finite) tree with root $T_{0}$. Be $n \in \mathbb{N}$ the number of objects in $L$, and $I:=\{1, \ldots, n\}$. A node corresponds to a sublist of $L$. In particular, $L=T_{0}$. $A$ node $t^{\prime}$ is a successor of node $t$, if $t^{\prime} \subseteq t$. For this we write $t^{\prime} \succ t$. If the list-inclusion is strict, we say that $t^{\prime}$ is an echt successor of $t$. For a list $l=$ $\left(t_{m}, \ldots, t_{0}\right)$ of nodes with $t_{m} \subseteq t_{m-1} \subseteq \ldots \subseteq t_{0}$, we set $m$ (the number of listinclusions) as length of $l$, and denote it with $\operatorname{lh}(l)$. With $\operatorname{slh}(l)$ we denote the number of strict list-inclusions. Also, we denote with $t^{\prime} \succ_{m} t$, that there is a list $l=\left(t, t_{1}, \ldots, t_{m-1}, t^{\prime}\right)$ with $\operatorname{lh}(l)=m$, and with $t^{\prime} \succ_{m}^{s} t$ the same situation with $\operatorname{slh}(l)=m$. The list-inclusions are governed by the principle of separation, that is, if, for $r \in \mathbb{N}, t^{\prime} \succ_{r}^{s} t$, then the objects of $t^{\prime}$ fulfill all the very same properties that the objects of $t$ fulfill, plus exactly $r$ more. If there is such a tree $T$ with $L=T_{0}$, and the union of all leaves gives $L$ again, we call it a distinction tree.

Definition 2 (Context). A context is a distinction tree with $L=E$, for some non-empty set $E$ of finite cardinality.

Now, if we evaluate (2), using (A $j)$ (with $j \in\{1, \ldots, 4\}$ ), and the context Vienna, where we take $C_{1}, \ldots, C_{k}$ as our comparison sets, i.e. we actually evaluate "Many (foreign) students (in Vienna) are German.", we have the following four results:

$j=1:(2)$ is true if and only if the number of German students in Vienna is at least as big as the number of students from $C_{i}$ in Vienna, for all $i \in$ $\{1, \ldots, k\}$.

$j=2$ : (2) is true if and only if the number of German students in Vienna is at least as big as the number of students from $C_{i}$ in Vienna, for more than half of the $i \in\{1, \ldots, k\}$. (Weakening w.r.t. $j=1$ )

$j=3:(2)$ is true if and only if the sum of the fractions that are smaller than the German one, is greater than the sum of fractions that are bigger than the German one. 
$j=4:(2)$ is true if and only if the fraction of German students in Vienna is greater than the one of every single comparison class. (Strengthening w.r.t. $j=1$ )

Note that, unlike later on in the intensional case, there is no need to introduce relativity counts here, since we would simply divide every term by the same normative term $\|A\|^{\text {sa }}(\neq 0)^{5}$, and hence it cancels out again immediately.

For readability and the sake of conciseness, we introduced only the four readings (A1),...,(A4), but we will now, as a preparation for Section 4, show, how the structure behind unfolds into a neat combinatorial pattern. Eventually, we intend to motivate the use of this pattern for a graded interpretation of many.

We can have the following 13 orders of the numbers $a, b$, and $c$ :

$$
\begin{array}{llll}
z_{1}: a>b>c & z_{2}: b>a>c & z_{3}: c>a>b & z_{4}: a=b>c \\
z_{5}: a>b=c & z_{6}: b>a=c & z_{7}: c>a=b & z_{8}: a=c>b \\
z_{9}: a>c>b & z_{10}: b>c>a & z_{11}: c>b>a & z_{12}: b=c>a \\
z_{13}: a=b=c & &
\end{array}
$$

These classes correspond to integer partitions where the order counts:

$$
\mathrm{IP}:=\left\{(a, b, c) \in \mathbb{N}^{3}: a+b+c=k\right\}
$$

We can make out three possible majority scenarios and one additional one :

(B1) for most $i \in\{1, \ldots, k\} p_{i}^{\mathrm{sa}}>p_{B}^{\mathrm{sa}}$

(B2) for most $i \in\{1, \ldots, k\} p_{i}^{\mathrm{sa}}=p_{B}^{\mathrm{sa}}$

(B3) for most $i \in\{1, \ldots, k\} p_{i}^{\text {sa }}<p_{B}^{\text {sa }}$

(B4) neither (B1) nor (B2) nor (B3) hold.

Of course, we could replace "most" with "all" in (B1), (B2), and (B3), but this would make considerations only easier, thus we decided to go for the slightly more involved (weaker) interpretation. Also, we want to point out, that the distinction into the four above scenarios will yield a more precise consideration than the one from (A1),..,(A4). It could easily be weakened to fit the former interpretation again, though. The reason why we are not doing it, is that we want to emphasize the inherent combinatorial systematics. Particularly, when we develop the graded interpretation of many, it will make sense to treat few simultaneously, and therefor we will need the full structure at our disposal. To

\footnotetext{
${ }^{5}$ Cf. remark on p. 10
} 
stress this a bit further, in [12] Lappin also attempts to so do as well. This can be read off the second quote of this paper, and from the following:

"A corresponding interpretation for \| few || is obtained by substituting || few $\|$

for $\|$ many $\|$ and '<' for ' $\geqslant$ ' in $(5)^{6}$."' ( [12], p. 601)

Singling out (B2) as a distinct set of scenarios will enable us to give an even more direct correspondence of the two determiners, in Section 4.

Clearly, $|\mathrm{IP}|=\frac{(k+1)(k+2)}{2}$.

The number of possible scenarios for (B1), (B2), and (B3) is the same, namely:

If $k$ is even: $\frac{1}{2}\left(\frac{k}{2}\right)\left(\frac{k}{2}+1\right)$. And if $k$ is odd: $\frac{1}{2}\left(\left\lfloor\frac{k}{2}\right\rfloor+1\right)\left(\left\lfloor\frac{k}{2}\right\rfloor+2\right)$.

The remaining scenarios from (B4) will be referred to as no-majority situations. Of them we have:

If $k$ is even: $\frac{1}{2}\left(\frac{k}{2}+1\right)\left(\frac{k}{2}+2\right)$. And if $k$ is odd: $\frac{1}{2}\left(\left\lfloor\frac{k}{2}\right\rfloor\right)\left(\left\lfloor\frac{k}{2}\right\rfloor+1\right)$.

Also, we could give a detailed analysis of the distribution of the orderings of the numbers $a, b$, and $c$, and show how they can be used to determine intermediate truth values of statements involving many. As this would take to much space, we delegate it to future work. Here, we only emphasize the existence of this pattern. The vicinity to probabilistic approaches (like the one of Fernando and Kamp) may be apparent, even from this narrow treatment, though. Still, we give an intuition how to evaluate (2) using more than two truth values, making use only of the numbers $a, b$, and $c$ :

$\operatorname{Many}(A, B)=0$, for scenarios from (B1) .

$\operatorname{Many}(A, B)=\frac{1}{2}$, for scenarios from (B2).

$\operatorname{Many}(A, B)=1$, for scenarios from (B3).

And for one of the remaining ones from (B4)

$c>a \rightarrow \operatorname{Many}(A, B) \in\left(\frac{1}{2}, 1\right], \quad$ and $\quad a>c \rightarrow \operatorname{Many}(A, B) \in\left[0, \frac{1}{2}\right)$

Later, we intend to look into that a little bit deeper and also make use of the measure of the corresponding comparison sets, as already indicated through (A3). Deeper considerations, into the direction of probability theory, can find a good starting point in [7].

\footnotetext{
${ }^{6}$ The (1) from the present paper is referred to as (5) in [12]
} 


\section{Diversification}

In this section, we will show, why Lappin's (1) is not enough to capture all readings of many, by giving an easy example, employing again (2). Before we will so do, lets fix some notation and have a look at the most apparent different interpretations: $\left(S=\left\{\mathrm{sn}_{i}: i \in\{1, \ldots, n\}\right\}\right)$

$p_{B, \text { rel }}^{\mathrm{sa}}:=\frac{\left|\|A\|^{\mathrm{sa}} \cap\|B\|^{\mathrm{sa}}\right|}{\left|\|A\|^{\mathrm{sa}}\right|} \quad$, and $\quad p_{B, \text { rel }}^{i}:=\frac{\left|\|A\|^{\mathrm{sn}_{i}} \cap\|B\|^{\mathrm{sn}_{i}}\right|}{|| A \|^{\mathrm{sn}_{i} \mid}}$, and

$p_{B, \text { abs }}^{\mathrm{sa}}:=\left|\|A\|^{\mathrm{sa}} \cap\|B\|^{\mathrm{sa}}\right|$, and $p_{B, \text { abs }}^{i}:=\left|\|A\|^{\mathrm{sn}_{i}} \cap\|B\|^{\mathrm{sn}_{i}}\right|$.

Be $I_{\alpha}:=\left\{i: i \in\{1, \ldots, n\}, p_{B, \text { rel }}^{i}>p_{B, \text { rel }}^{\mathrm{sa}}\right\} \quad$, and $\alpha:=\left|I_{\alpha}\right|$.

Be $I_{\beta}:=\left\{i: i \in\{1, \ldots, n\}, p_{B, \text { rel }}^{i}=p_{B, \text { rel }}^{\text {sa }}\right\} \quad$, and $\beta:=\left|I_{\beta}\right|$.

Be $I_{\gamma}:=\left\{i: i \in\{1, \ldots, n\}, p_{B, \text { rel }}^{i}<p_{B, \text { rel }}^{\text {sa }}\right\} \quad$, and $\gamma:=\left|I_{\gamma}\right|$.

Be $I_{\tilde{\alpha}}:=\left\{i: i \in\{1, \ldots, n\}, p_{B, \text { abs }}^{i}>p_{B, \text { abs }}^{\mathrm{sa}}\right\} \quad$, and $\tilde{\alpha}:=\left|I_{\tilde{\alpha}}\right| \cdot$

Be $I_{\tilde{\beta}}:=\left\{i: i \in\{1, \ldots, n\}, p_{B, \text { abs }}^{i}=p_{B, \text { abs }}^{\mathrm{sa}}\right\} \quad$, and $\tilde{\beta}:=\left|I_{\tilde{\beta}}\right|$.

Be $I_{\tilde{\gamma}}:=\left\{i: i \in\{1, \ldots, n\}, p_{B, \text { abs }}^{i}<p_{B, \text { abs }}^{\mathrm{sa}}\right\} \quad$, and $\tilde{\gamma}:=\left|I_{\tilde{\gamma}}\right|$.

$$
\begin{aligned}
& \|B\|^{\text {sa }} \in \| \text { many } \|\left(\|A\|^{\text {sa }}\right) \text { iff } \\
& \qquad S \neq \emptyset \text {, and for every } i \in\{1, \ldots, n\}, \text { it holds: } p_{B, \text { rel }}^{\text {sa }} \geqslant p_{B, \text { rel }}^{\mathrm{i}}
\end{aligned}
$$

Other options are striking, like the following two weakenings:

$$
\begin{aligned}
& \|B\|^{\mathrm{sa}} \in \| \text { many } \|\left(\|A\|^{\mathrm{sa}}\right) \text { iff } \\
& \qquad \begin{array}{l}
S \neq \emptyset, \text { and for most } i \in\{1, \ldots, n\}, \text { it holds: } p_{B, \text { abs }}^{\mathrm{sa}} \geqslant p_{B, \text { abs }}^{\mathrm{i}} \\
\|B\|^{\mathrm{sa}} \in \| \text { many } \|\left(\|A\|^{\mathrm{sa}}\right) \text { iff } \\
\qquad S \neq \emptyset, \text { and for most } i \in\{1, \ldots, n\}, \text { it holds: } p_{B, \text { rel }}^{\mathrm{sa}} \geqslant p_{B, \text { rel }}^{\mathrm{i}}
\end{array}
\end{aligned}
$$

Or similarly to what we did before, we can use these two strengthenings:

$$
\begin{aligned}
& \|B\|^{\mathrm{sa}} \in \| \text { many } \|\left(\|A\|^{\mathrm{sa}}\right) \text { iff } \\
& \qquad S \neq \emptyset, \text { and it holds: } p_{B, \mathrm{abs}}^{\mathrm{sa}}>\max \left\{p_{B, \mathrm{abs}}^{i}: i \in\{1, \ldots, n\}\right\}
\end{aligned}
$$




$$
\begin{aligned}
& \|B\|^{\mathrm{sa}} \in \| \text { many } \|\left(\|A\|^{\mathrm{sa}}\right) \text { iff } \\
& \qquad S \neq \emptyset, \text { and it holds: } p_{B, \text { rel }}^{\mathrm{sa}}>\max \left\{p_{B, \text { rel }}^{i}: i \in\{1, \ldots, n\}\right\}
\end{aligned}
$$

To complete our selection:

$$
\begin{aligned}
& \|B\|^{\mathrm{sa}} \in \| \text { many } \|\left(\|A\|^{\mathrm{sa}}\right) \text { iff } \\
& \qquad S \neq \emptyset \text {, and it holds: } \sum_{i \in I_{\tilde{\gamma}}} p_{B, \text { abs }}^{i}>\sum_{i \in I_{\tilde{\alpha}}} p_{B, \mathrm{abs}}^{i} \\
& \|B\|^{\mathrm{sa}} \in \| \text { many } \|\left(\|A\|^{\mathrm{sa}}\right) \text { iff } \\
& \qquad \begin{array}{l}
S \neq \emptyset, \text { and it holds: } \sum_{i \in I_{\gamma}} p_{B, \mathrm{rel}}^{i}>\sum_{i \in I_{\alpha}} p_{B, \mathrm{rel}}^{i}
\end{array}
\end{aligned}
$$

Remark: To not run into trouble here, we use the following common interpretation: For two sets $M$ and $N$, we have: $\frac{|M \cap N|}{|N|}=0$, if $|N|=0$.

A natural question is now, whether we really do something new here, or whether there is a way to translate (3)-(9), by some means, into (1). This can also be so expressed:

Proposition 1. Let $S=\left\{\mathrm{sn}_{1}, \ldots, \mathrm{sn}_{n}\right\}$ be a set of normative situations. Furthermore, assume we want to employ the relative reading of many captured by (3). It is not possible to rewrite $S$ into $S^{\prime}=\left\{\mathrm{sn}_{1}^{\prime}, \ldots, \mathrm{sn}_{n}^{\prime}\right\}$ such that $S^{\prime}$ is a set of normative situations, and we can employ (1) equivalently to (3) using this $S^{\prime}$ instead of $S$.

Proof. Assumptions:

- $\|A\|^{\text {sa }} \neq \emptyset$, and $\|A\|^{\text {sn }} \neq \emptyset$ for all sn $\in S$

- $\forall_{\mathrm{sn} \in S}: \quad\left|\|A\|^{\text {sa }} \cap\|B\|^{\text {sa }}\right| /\left|\|A\|^{\text {sa }}\right| \geqslant\left|\|A\|^{\text {sn }} \cap\|B\|^{\text {sn }}\right| /\left|\|A\|^{\text {sn }}\right|$

We are looking for a $S^{\prime}$ such that:

$\forall_{\mathrm{sn}^{\prime} \in S^{\prime}}: \quad\left|\|A\|^{\mathrm{sa}} \cap\|B\|^{\mathrm{sa}}\right| \geqslant\left|\|A\|^{\mathrm{sn}^{\prime}} \cap\|B\|^{\mathrm{sn}^{\prime}}\right|$

Our setting grants us, that $A$ and $B$ are fixed predicates. Also, we can fix some sn from $S$ for which we have equality in $(\otimes)$, and try to rewrite it into an $\mathrm{sn}^{\prime}$, while sa is fixed from the very beginning. Thus, generally by assumption, $q:=\frac{\|A\|^{\mathrm{sa}}}{\|A\|^{\mathrm{sn}}}$ is a non-negative rational number, depending particularly on the actual situation 
sa. Hence, it can be, that $q=\frac{1}{3}$, as a special case, as well as it may be the case, that $\left|\|A\|^{\mathrm{sn}} \cap\|B\|^{\mathrm{sn}}\right|=26$. Now, since $\left|\|A\|^{\mathrm{sn}^{\prime}} \cap\|B\|^{\mathrm{sn}^{\prime}}\right| \in \mathbb{N}$, it can not be, that:

$$
\|\| A\left\|^{\mathrm{sn}} \cap\right\| B\left\|^{\mathrm{sn}}\left|\frac{\|A\|^{\mathrm{sa}}}{\|A\|^{\mathrm{sn}}}=\right|\right\| A\left\|^{\mathrm{sn}^{\prime}} \cap\right\| B \|^{\mathrm{sn}^{\prime}} \mid
$$

since that would mean, that $\frac{26}{3} \in \mathbb{N}$, which is clearly wrong. This completes the argument.

(4) can be treated differently. Indeed, we can switch to a subset $S^{\prime}$ of $S$ for which we have that, (4) becomes (1), if we replace $S$ by $S^{\prime}$. Regarding (5), (7) and (9), we can use a similar argument like for $(3)$, hence they are not translatable into (1). (6) is just a strengthening of (1), thus, the first implies the latter, but not the other way around. Hence, if we start off with (1), we can rewrite the $S$ from there into a $S^{\prime}$, by dropping the situations for which we have equality, and arrive at (6). Since (8) is a new plausible reading of many, not directly referring to $p_{B \text {,abs }}^{\mathrm{sa}}$ at all, it is not translatable into (1) without losing the intended information that it should express.

This shows, that there is much more to achieve than (1) suggests. Now, again for clarity, lets look again at (2) and evaluate it with regard to the following set of normative situations $S=\left\{\mathrm{sn}_{1}, \mathrm{sn}_{2}, \mathrm{sn}_{3}\right\}$, with $\mathrm{sn}_{1}=$ New York,

$\mathrm{sn}_{2}=$ London, and $\mathrm{sn}_{3}=$ Paris. So, what we intend to evaluate is, actually, the following statement:

"Many students are German (in Vienna, compared to NY, London and Paris)".

Lets consider the following fictive numbers ${ }^{7}$ :

City $\quad$ inhabitants $\mid$ German students $\mid$ per cent $\mid$ students total|relative count

\begin{tabular}{l|c|c|c|c|c}
\hline Wien & 1800000 & 20000 & $\sim 0.011$ & 60000 & $\sim 0.33$ \\
\hline NY & 10000000 & 20010 & $\sim 0.002$ & 150000 & $\sim 0.13$ \\
\hline London & 10000000 & 12000 & $\sim 0.0012$ & 100000 & $\sim 0.12$ \\
\hline Paris & 2200000 & 10010 & $\sim 0.0046$ & 80000 & $\sim 0.125$
\end{tabular}

Using (1), we are bound to evaluate (2) to false, unlike with, say (3), since, even though there is a normative situation in which we have (absolutely) more German students than in the actual one, it is still true, that Vienna has (relatively) the most German students within all normative situations. This is still a plausible reading of many. Since (3) is just the relative version of (1), it it obvious how the interpret the two ( absolute and relative) weakenings (4) and (5), as well as the two (absolute and relative) strengthenings (6) and (7). Clearly, (8) and (9) are the truly new intensional readings of many. Again, we point out, that, unlike before in the extensional case, here it makes a difference whether we introduce

\footnotetext{
7 'German students' corresponds to $|\|B\| \cap\|A\||$, 'students' corresponds to $|\|A\||$, and
} 'relative count' corresponds to $\frac{|\|B\| \cap\|A\||}{|\|A\||}$. 
a relative version of (8), namely (9), for the normative situations are distinct. These respectively evaluate to:

W.r.t. (8): $\operatorname{Many}(A, B)=1$, since $10010+12000>20010$

W.r.t. (9): $\operatorname{Many}(A, B)=1$, since $0.12+0.125+0.13>0$

\section{Fuzzy Range}

In this section, we intend to arrive at first options for interpreting many in the semy-fuzzy case, i.e. we want our predicates to be crisp, and classically interpreted, but at the same time, we want to take up the challenge of graded interpretations of many. In Glöckner's [8], no intensionality is considered.

Lets have a look at the following function:

$f: \mathbb{Q}_{0}^{+} \times \mathbb{Q}_{0}^{+} \backslash\{0,0\} \longrightarrow[0,1]$, with $f(x, y)=\frac{x}{x+y}$

This one fulfills the following five properties:

- $x=y$ implies, that $f(x, y)=\frac{1}{2} \quad \bullet x>y$ implies, that $f(x, y) \in\left(\frac{1}{2}, 1\right]$

- $x<y$ implies, that $f(x, y) \in\left[0, \frac{1}{2}\right) \quad \bullet y=0$ implies, that $f(x, y)=1$

- $x=0$ implies, that $f(x, y)=0$

Thus, as a first option one can give the following refinement, for scenarios from (B4), of what we did at the end of Section 2:

$\operatorname{Many}(A, B)=f(c, a)=\frac{c}{c+a}$

A deeper consideration (still with regard to the extensional case) may be this one:

$\operatorname{Many}(A, B)=f\left(\sum_{i \in I_{c}} p_{i}^{\mathrm{sa}}, \sum_{i \in I_{a}} p_{i}^{\mathrm{sa}}\right)=\frac{\sum_{i \in I_{c}} p_{i}^{\mathrm{sa}}}{\sum_{i \in I_{c}} p_{i}^{\mathrm{sa}}+\sum_{i \in I_{a}} p_{i}^{\mathrm{sa}}}$

The combinatorial structure used above allows for an analogue treatment of the intensional case:

(C1) for most $i \in\{1, \ldots, n\} p_{B, \text { rel }}^{i}>p_{B \text {,rel }}^{\text {sa }}$

(C2) for most $i \in\{1, \ldots, n\} p_{B, \text { rel }}^{i}=p_{B, \text { rel }}^{\text {sa }}$

(C3) for most $i \in\{1, \ldots, n\} p_{B, \text { rel }}^{i}<p_{B, \text { rel }}^{\text {sa }}$

(C4) neither (C1) nor (C2) nor (C3) hold. 
Thus (for scenarios from $(\mathrm{C} 4)$ ):

W.r.t.(5):

$$
\operatorname{Many}(A, B)=f(\gamma, \alpha)=\frac{\gamma}{\gamma+\alpha}
$$

W.r.t.(9):

$$
\operatorname{Many}(A, B)=f\left(\sum_{i \in I_{\gamma}} p_{B, \text { rel }}^{i}, \sum_{i \in I_{\alpha}} p_{B, \text { rel }}^{i}\right)=\frac{\sum_{i \in I_{\gamma}} p_{B, \text { rel }}^{i}}{\sum_{i \in I_{\gamma}} p_{B, \text { rel }}^{i}+\sum_{i \in I_{\alpha}} p_{B, \text { rel }}^{i}}
$$

And for the remaining ones, analogously to the extensional case:

$\operatorname{Many}(A, B)=0$, for scenarios from $(\mathrm{C} 1)$.

$\operatorname{Many}(A, B)=\frac{1}{2}$, for scenarios from $(\mathrm{C} 2)$.

$\operatorname{Many}(A, B)=1$, for scenarios from $(\mathrm{C} 3)$.

Note, that for all scenarios from (B4) and (C4) it is not possible for $a$ and $c$, or $\alpha$ and $\gamma$ respectively, to be zero at the same time. In fact, only if $k$, or $n$ respectively, is even, it may happen, that one of them is zero. Otherwise both values are bound to be greater than zero.

So, through singling out the scenarios (B2) and (C2), we can now see the symmetry of the parameters $a$ and $c$, or $\alpha$ and $\gamma$ respectively. This gives us the opportunity to define few complementary to many, by just swapping the denotation of the respective values $a$ and $c$, or $\alpha$ and $\gamma$ respectively. This is also an argument why one may prefer the strict inequalities over the non-strict ones, and at the same time a singling out of the third case to which we assigned the intermediate truth value $\frac{1}{2}$. A full characterization of few is not part of this paper, but can be carried out straightforwardly, particularly by means of this functional equality:

$\operatorname{Few}(A, B)=1-\operatorname{Many}(A, B)$

\section{Conclusion and Future Work}

We have seen how Lappin's parametrization of many provides a neat frame to evaluate natural language statements involving vague quantifier expressions. On top of that, we augmented the applicability through giving additional plausible readings, for both the extensional and the intensional case. We have seen how combinatorics can facilitate considerations, but interesting parts had to be delegated to future work. The full characterization of all mentioned distributions is clearly our attempt, as well as formally making out all relevant parameters in play. One class of readings that we did not touch in this paper are the subjective ones. For example, further information about our comparison classes, such 
as topological distance, and even sympathy values, can enter the setting. How to rank these evaluation techniques is a very interesting question, that we will pursue in the near future. Game semantics, in particular with more than two players, will surly play an important role. This will bring us to the true fuzzy scenario, in which the predicates will not necessarily be classically interpreted anymore. Also, of course, we will treat more complex statements involving logical fuzzy connectives such as $\wedge, \vee$.

\section{References}

1. J. Barwise and R. Cooper. Generalized quantifiers and natural language. Linguistics and philosophy, 4(2):159-219, 1981.

2. P. Cintula, P. Hájek, and C. Noguera, editors. Handbook of Mathematical Fuzzy Logic. College Publications, 2011.

3. C.G. Fermüller and C. Roschger. Randomized game semantics for semi-fuzzy quantifiers. In S. et.al. Greco, editor, Advances in Computational Intelligence, volume 300, pages 632-641. Springer, 2012.

4. C.G. Fermüller and C. Roschger. Bridges between contextual linguistic models of vagueness and t-norm based fuzzy logic. In Franco Montagna, editor, Petr Hájek on Mathematical Fuzzy Logic, volume 6, pages 91-114. Springer, 2014.

5. C.G. Fermüller and C. Roschger. Randomized game semantics for semi-fuzzy quantifiers. Logic Journal of the IGPL, 223(3):413-439, 2014.

6. T. Fernando and H. Kamp. Expecting many. In Semantics and Linguistic Theory, pages 53-68, 1996.

7. P. Flajolet and R. Sedgewick. Analytic Combinatorics. Cambridge University Press, 2009.

8. I. Glöckner. Fuzzy quantifiers: A computational theory, volume 193 of Studies in Fuzziness and Soft Computing. Springer Verlag, 2006.

9. E. L. Keenan and J. Stavi. A semantic characterization of natural language determiners. Linguistics and Philosophy, 9(3):253-326, 1986.

10. S. Lappin. The semantics of "many" as a weak determiner. Linguistics, 26(6):9771020, 1988.

11. S. Lappin. Many as a two-place determiner function. SOAS working papers in linguistics and phonetics, 3:337-358, 1993.

12. S. Lappin. An intensional parametric semantics for vague quantifiers. Linguistics Es Philosophy, 23(6):599-620, 2000.

13. S. Peters and D. Westerståhl. Quantifiers in Language and Logic. Oxford University Press, USA, 2006.

14. D. Westerståhl. Quantifiers in formal and natural languages. In Handbook of philosophical logic, pages 1-131. Springer, 1989.

15. L. A. Zadeh. A computational approach to fuzzy quantifiers in natural languages. Computers $\mathcal{E}$ Mathematics with applications, 9(1):149-184, 1983. 